BAL 51602

UC-28

(Particle Accoierators and

High-Voltage Whehines - TiC-4500)

BNL -51602

DE83 008470

\title{
AN e-p PRIMER
}

\author{
D. Hywel White
}

\section{October 15, 1982}

\author{
DISCLAIMER
}

\begin{abstract}
This report was prepared as an account of work sponsored by an agency of the United States Government. Neither the United States Government nor any agency thereof, nor any of their employees, makes any warranty, express or implied, or assumes any legal liability or responsibility for the accuracy, completeness, or usefulness of any information, apparatus, product, or process disclosed, or represents that its use would not infringe privately owned rights. Reference herein to any specific commercial product, process, or service by trade name, trademark, manufacturer, or otherwise does not necessarily constitute or implv its endorsement, recommendation, or favoring by the United States Government or any agency thereof. The views and opinions of authors expressed herein do not necessarily state or reflect those of the United States Government or any agency thereof.
\end{abstract}

\section{ACLELERATOR DEPARTMENT}

\author{
BROOKHAVEN NATIONAL LABORATORY \\ ASSOCIATED UNIVERSITIES, INC. \\ UPTON, LONG ISLAND, NEW YORK 11973
}




\section{DISCLAIMER}

This report was prepared as an account of work sponsored by an agency of the I'nited States Government. Neither the I'nited States Government nor any agency thereof. nor any of their employees. nor any of their contractors, subsontractors, or their employees makes any warranty, express or implied, or assumes any legal liability or responsibility for the acruracy, completeness, or usefulness of any information. apparatus, product, or process disclosed. or represens that its use would not intringe privately owned rights. Reference herein to any specific commercial product, process, or service by Irade name, Irademark, manufacturer, or otherwise, does not necessarily ronstitute or imply its endorsement, recommendation. or lavoring by the IInited States Government or any agency. contrartor or subcontractor thereof. The views and opinions of authors expressed herein do not necessarily state or reflect those of the I nited States Government or any agency. contractor or subcontractor thereof.

Printed in the Inited States of America Available from

National Technical Information Service

U.S. Department of Commerce

5285 Port Royal Road

Springfield, VA 22161

NTIS price codes:

Printed Copy: A02; Microfiche Copy: A0I

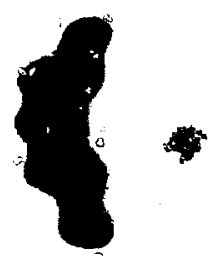




\begin{abstract}
The basic formulas for kinematics and calculating yields of events for an e-p collider are presented, together with typical results.
\end{abstract}


An e-p Primer

D. Hywel thite

The interest in e-p colliders in recent years has inspired a series of studies, frequently designed as an add-on to other existing facilities. In our experience, Bjorn Wilk has promoted this field of physics for many years, and his efforts have culminated in a proposal for HERA. In the U.S., the Columbia Group, the Canadians, and collaborators have recently proposed electron rings as a complement to proton facilities at Fermflab and Brookhaven. We have listed in refs. 1 to 4 the reports that marked the development of this concept in high energy physics. The physics is sometimes held to be self-evident, an extension of the work on structure functions at SLAC and later at Fermilab and CERN, and also of the elegant demonstration of the existence of the weak neutral current with polarized electrons at SLAC. Although this field is not new, the basic ideas often seem to be poorly understood by both the protagonists and the antagonists of e-p physics. This paper is inspired by a task force at Brookhaven charged with a review of the feastbility of operating an e-p collider on that site specifically, to capitallze on the local advantages. 5 The DPF Summer study group was also asked to consider the physics of $e-p$ in a more general way, and we offer this note fresh from relearning the kinematics and the specific predictions of the standard model for event rates with an e-p collider.

In reviewing the extensive literature, it became clear that simpleminded relations that allowed comparison between options were not avallable. Each report has concentrated on a specific combination of electron and proton energies. We have trled, therefore, to generate rules of thub which will allow comparison of options for future consideration, and we attempt to make available some appropriate simplifications which may allow others to reach their own judgments on the merits of varlous comblnations.

First, then, we discuss the kinematics, which tend to be rather different from those at fixed target machines and symmetric colliders. of course, we make use of invariants whenever posstble, with an attempt to make clear thelr effect in a laboratory situation. We have selected three invariants as our prime cholce to describe the scattering processes: $s$ (the c.m. energy squared), $Q^{2}$ (the negative four-momentum transfer squared to to the electron), and $x$ (the fraction of the target momentum carried by the struck parton). We will make connections to the other frequently used variables $y, v$, and $W$, the $c . m$, energy of the photon (boson), proton system.

\section{Kinematics}

We subscript the proton varlables with a $p$ and leave electron variables unsubscripted. The c.m. energy squared is given as

$$
s=\left(E+E_{p}\right)^{2}-\left(\vec{p}-\vec{p}_{p}\right)^{2},
$$


where $\vec{p}_{p}$ is opposite to $\vec{p}$ in the lab system; then neglecting the mass of the electron,

$$
s=2 E E_{p}+2 E P_{p}+q_{p}^{2} \text {. }
$$

At the energies appropriate to this discussion, we should neglect terms $\sim\left(1 / E_{p}\right)$ giving

$$
s=4 E E_{p}+q_{p}^{2} \sim 4 E_{1} E_{p}
$$

The four momentum transfer squared

$$
\begin{aligned}
q^{2} & =\left(E-E^{\prime}\right)^{2}-\left(E-E^{\prime} \cos \theta\right)^{2}-E^{\prime} \sin ^{2} \theta, \\
& =-2 E E^{\prime}(1-\cos \theta) .
\end{aligned}
$$

It is customary to use $Q^{2}=q^{2}-4 E E^{\prime} \sin ^{2}(\theta / 2)$. This variable is used as the scale-breaking parameter in the hadronic structure functions as we shall see below. The third variable is $x$, the fraction of the hadron momentun that is carried by the struck parton. Before we define $x$, we define $v$ :

$$
v=\frac{\mathbb{P} \cdot q}{\mathbb{G}_{p}}
$$

$\mathbb{P}$ is the four-momentum of the proton and $q$ is the four momentum transfer to the electron. The invariant is especially simple in fixedtarget physics when $P=\left(m_{p}, 0\right)$ and then $v=E-E^{\prime}$. In the collider,

$$
\begin{aligned}
P . q & =E_{p}\left(E-E^{\prime}\right)+P_{p}\left(E-E^{\prime} \cos \theta\right) \\
& =2 E E_{p}-E^{\prime} E_{p}(1+\cos \theta) .
\end{aligned}
$$

This has a maximum value when $\theta=\pi$ :

then

$$
v_{\max }=\frac{2 E E_{p}}{m_{p}} \sim s / 2 m_{p} \text {; }
$$

$$
y=v / v_{\max }=\frac{2 p \cdot q}{s},
$$


and the third variable

$$
x=\frac{Q^{2}}{2 \mathbb{P} \cdot q}=\frac{Q^{2}}{\text { sy }}
$$

Both dimensionless varlables $x$ and $y$ go from 0 to 1 .

Although we calculate rates as a function of $s, Q^{2}$, we often use cross sections expresser as a function of $s, x$, and $y$; then

and

$$
y=Q^{2} / s x
$$

$$
\frac{d}{d y}=s \times \frac{d}{d Q^{2}} \text {. }
$$

Much of the physics of an e-p collider is reached by measuring the ourgolng electron; we now discuss this facet of the kinematics. In the basic model of the Interaction, elastic scattering occurs between the electron and a parton which carries a fraction $x$ of the Incoming nucleon momentum, as in the diagram below:

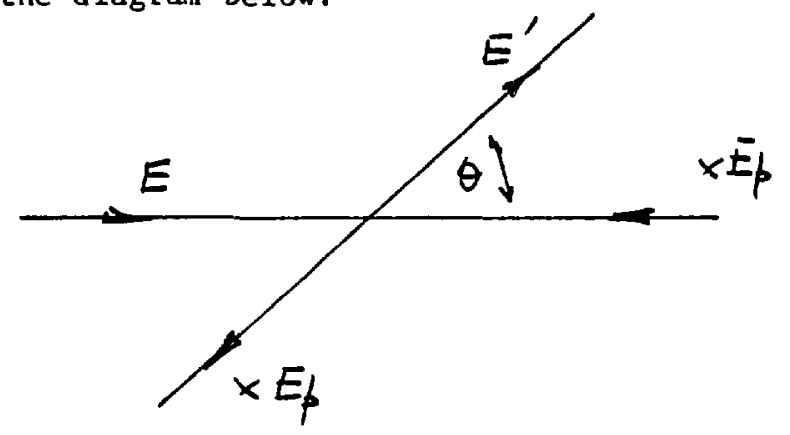

We still have $Q^{2}=2 E^{\prime}(1-\cos \theta)$, and

$$
y=\frac{Q^{2}}{s x}=\frac{2 E E^{\prime}(1-\cos \theta)}{4 E E_{p} x}
$$

so

$\Xi_{p}$ is the energy of the proton in the lab system and $E=E^{\prime}=x_{P}$ '

$$
y=\frac{1}{2}(1-\cos \theta) \text {, }
$$

and

$$
(1-y)=\frac{1}{2}(1+\cos \theta) \text {. }
$$


We understand the significance of $(1-y)$ as follows. When $y=1$, the electron is scattered at $180^{\circ}$; for a given helicity of the incident electron, the exchanged photon (boson) is completely polarized. The struck quark must change helicity by one unit to absorb the photon (boson) and so only quarks of the appropriate helicity can interact. This term $(1-y)$ appears in the cross sections for the part where the quark helicity is important.

In the laboratory system of the collider, the locus of the momentum vector is an ellipse with the interaction region at one focus as shown below.

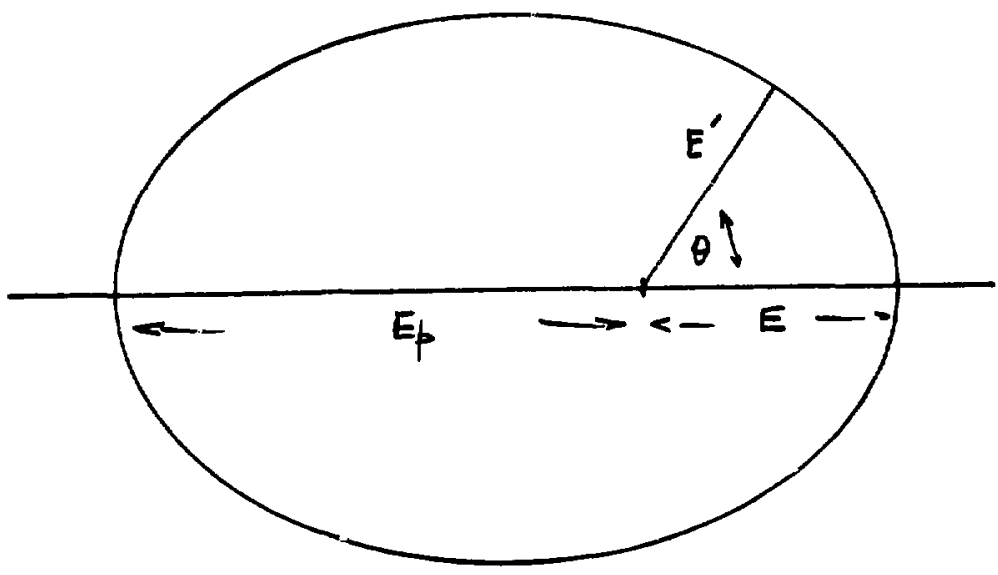

When $x=1$, the electron momentum is maximal for any given outgoing angle and the equation of the ellipse is

$$
\frac{1}{E^{\prime}}=\alpha(1+\varepsilon \cos \theta) \text {, }
$$

with

$$
\varepsilon=\frac{E_{p}-E}{E_{p}+E},
$$

and

$$
\alpha=\frac{E_{p}+E}{2 E E_{p}} \text {. }
$$


When $x \neq 1$, then the locus is still an ellipse but with $\mathrm{E}_{\mathrm{p}}$ replaced by $x E_{p}$, which gives

$$
\frac{1}{E^{\prime}}=\frac{\left(x E_{p}+E\right)}{2 E x E_{p}}\left(1+\frac{x E_{p}-E}{x E_{p}+E} \cos \theta\right)
$$

At $\theta=0$ and $\pi, E^{\prime}$ is $E$ and $x_{p}$ as expected. Using the ellipse equation, we can write

$$
x=\frac{E E^{\prime}(1-\cos \theta)}{E_{p}\left[2 E^{\prime}-E^{\prime}(1+\cos \theta)\right]}
$$

Since we measure $\theta$ and $E^{\prime}$, we can also write

$$
\frac{d x}{x}=\frac{1}{2}\left[1+\frac{x E_{p}}{E}\left(\cot ^{2} \theta / 2\right)\right] \frac{d E^{\prime}}{E^{\prime}} \text {. }
$$

When $\theta=0$, this factor becomes infinite and so we know $E^{\prime}$ and $x$ are decoupled. Any knowledge of $x$ comes from the current jet. As we go away from $\theta=0$, the term $\left(x E_{p} / E \cot ^{2}(\theta / 2)\right.$ is still troublesome if we have $a$ collider with $x E_{p} \gg E$ for interesting values of $x$. We can get around this with measurements of the current jet again but with reduced resolution.

In addition, all quasi-photoproduction is done at low $Q^{2}$, and although $E^{\prime}$ becomes smaller than $E$ and energy is transferred to the virtual phricon, the mass of the current jet (onia) cannot be determined from the outgoing electron.

When the electron energy is very low compared to the proton energy, the outgolng electron is scattered over a wider angle for a given $Q^{2}$. For example, $Q^{2}=4 E^{2}$ at $\theta=90^{\circ}$ so the $Q^{2}$ range that is accessible in the forward electron hemisphere depends directly on the incident electron energy. It is useful for experimental reasons to concentrate the outgoing cone of useful electrons and thus keep the electron energy as high as possible. 


\section{The Electromagneitc Process}

The leading term in inelastic electron scattering is shown in the following diagram.

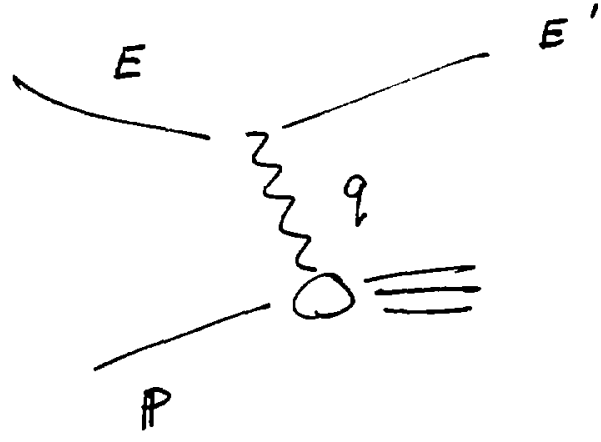

The cross section for this process is often written

$$
\frac{d^{2} \sigma}{d x d y}=\frac{4 \pi \alpha^{2}}{s x^{2} y^{2}}\left[(1-y) F_{2}\left(x, Q^{2}\right)+y^{2} \times F_{1}\left(x, Q^{2}\right)\right] .
$$

The Callan-Gross relation is derived from the assumption that partons (quarks) that couple to photons have $\operatorname{spin} 1 / 2$ and gives $F_{2}=2 \times F_{1}$ and then

$$
\frac{\mathrm{d} \sigma^{2}}{\mathrm{dxd} Q^{2}}=\frac{4 \pi \alpha^{2}}{x Q^{4}}\left[1-\frac{Q^{2}}{s x}+\frac{1}{2}\left(\frac{Q^{2}}{s x}\right)^{2}\right] F_{2}\left(x, Q^{2}\right) \text {. }
$$

The function in the square brackets is 1 when $Q^{2}=0$ and $1 / 2$ when $Q^{2}$ is the maximum for that $s$ and $x$. The variation is monotonic between these bounds and, as we will see below, at the values of $Q^{2}$ that are accessible, this function varies slowly and is always close to unity. This leads us to the assertion that if the values of $Q^{2}$ and $x$ are accessible to the kinematic region, then the cross section is almost independent of $s$.

We have the prejudice that much of the physics basic to electroproduction involves the detailed measurement of distributions with reasonable precision in each bin. We have chosen bins that are constant in $\mathrm{dQ}^{2} / \mathrm{Q}^{2}$ and $\mathrm{dx} / \mathrm{x}$. We choose $\mathrm{dQ}^{2} / \mathrm{Q}^{2}$ because the scale-breaking effects are logarithmic in $Q^{2}$, and so at large $Q^{2}$ we can afford to use larger bins. In contrast, the most rapid variations in the structure functions are at 
low $x$ and thus, to observe the functions properly, we need fine bins at low $x$. We have chosen in our plot $d^{2} / Q^{2}=0.1$ and $d x / x=0.1$ aiso. If these bins are seen to be too fine (or coarse), then the rate adjustment is trivial. We must discuss luminosities at some length, for, as we see below, they are crucial, but for the moment we assume that an integrated luminosity for a single experimental configuration of $10^{38}$ is a reasonable number.

Then the rate in each bin is

$$
\operatorname{RATE}=\frac{4 \pi \alpha^{2}}{Q^{2}}\left[1-\frac{Q^{2}}{x s}+\frac{1}{2}\left(\frac{Q^{2}}{x s}\right)^{2}\right] F_{2}\left(x, Q^{2}\right) \frac{d Q^{2}}{Q^{2}} \frac{d x}{x} .
$$

Numerically, $Q^{2}$ is in $\mathrm{GeV}^{2}$, as is $s$ and then

$$
4 \pi \alpha^{2}=4 \pi(1 / 137)^{2} 0.197^{2} \times 10^{-26}=2.60 \times 10^{-31} \mathrm{~cm}^{2} \mathrm{GeV}^{2} .
$$

For the assumed bin width and integrated luminosity of $10^{38}$,

$$
\operatorname{RATE}=\frac{2.60 \times 10^{5}}{Q^{2}}\left[1-\left(\frac{Q^{2}}{x s}\right)+\frac{1}{2}\left(\frac{Q^{2}}{s x}\right)\right] F_{2}\left(x, Q^{2}\right) .
$$

In very approximate terms, the photon couples to the charge squared of the quark, so that it is four times as likely to see an up quark as a down. Thus at any finite $x$, we can neglect the sea and guess that $F_{2}$ $\sim 2 / 3$. At $Q^{2}=1000$, we get 170 events in a bin. A real calculation give 104 which is a pretty fair agreenent. Frequently the rate above a given $Q^{2}$ is plotted which gives a ilatter dependence on $Q^{2}$ which we feel is not especially relevant to the physics of nucleon structure. We have arbitrarily assumed that 100 events in our chosen bin are our threshold of measurement credibility. We can then make a curve which traces the location of this limiting bin as a function of $x$ and $Q^{2}$ for a given $x$. We show such a plot in Figure 1. We Include weak neutral current effects here, but note that the single photon exchange process falls between the $e_{\mathrm{L}}^{-i}$ and $e_{\mathrm{L}}^{t}$ curves. $s$ is chosen at $40,000 \mathrm{GeV}^{2}$ ( 25 on 400). First note that the kinematic boundary is way off scale $\left(0_{\text {max }}^{2}=s=40,000\right)$. Our first lesson is that the region of accessible measurements is luminosity linited and nct s limited. The line ntar $x=0$ is the kinematic limit and although the low $x$ high $Q^{2}$ region is limited by $s$, it it is only slightly so.

In Figure 2, we show a similar curve with $s=32,000$ but with slightly improved luminosity, and we see that luminosity wins. It seems a shame to ignore the events above the luminosity boundary and we offer an approximate method for computation. Note first that $F_{2}\left(x, q^{2}\right)$ varies quite slowly with $Q^{2}$ and we can ignore that varlation 


$$
\operatorname{RATE}=1000 \mathrm{Q}_{100}^{2} \int_{\mathrm{Q}_{100}^{2}}^{\mathrm{sx}} \frac{\mathrm{dQ}^{2}}{\mathrm{Q}^{4}}
$$

$=1000\left(1-Q_{100}^{2} / s x\right)$

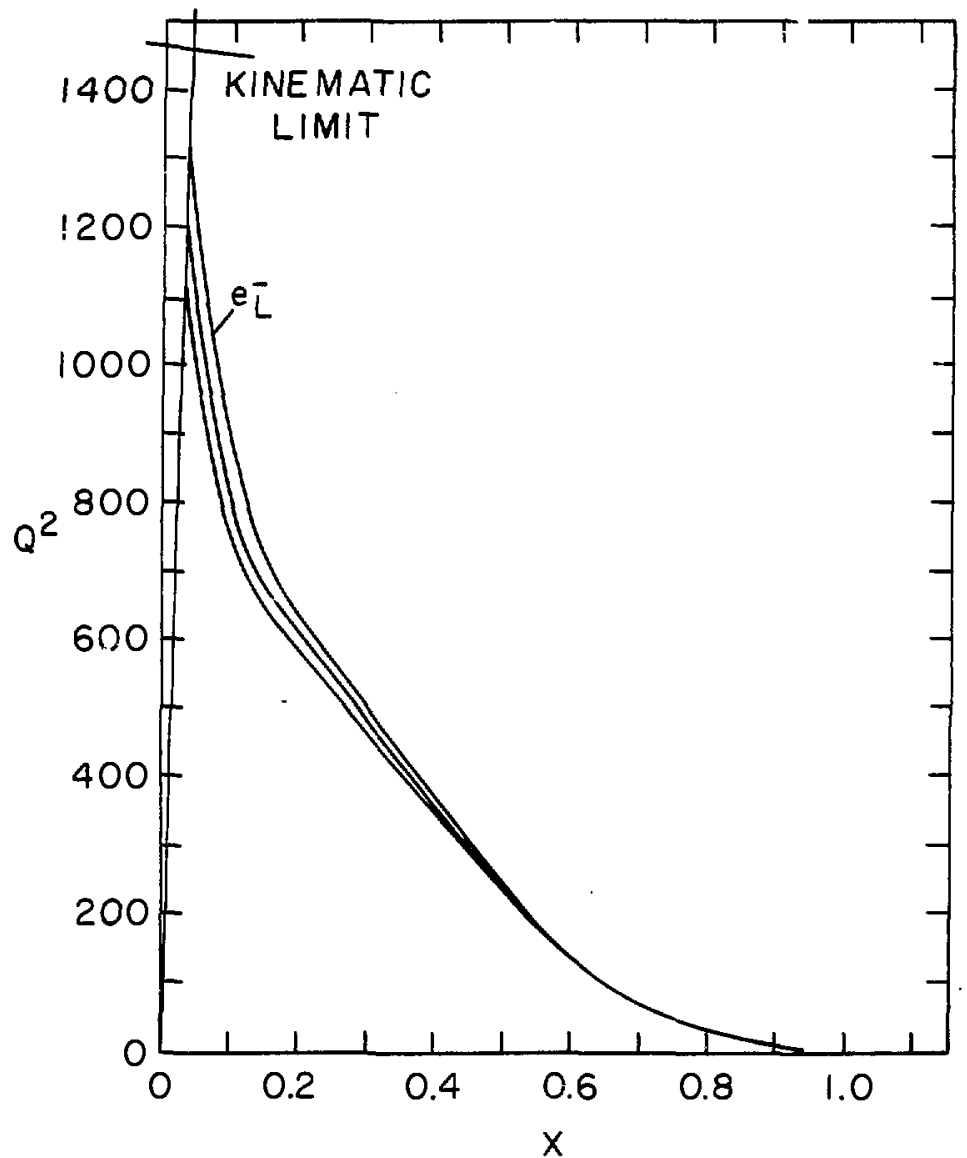

Fig. 1. The luminosity boundary for the reaction $e=p+e^{\prime}+x$ for $s$ $=40,000 \mathrm{GeV}^{2}, \sin ^{2} \theta_{\mathrm{w}}=0.22$, and an integrated luninosity of $10^{38} \mathrm{~cm}^{-2}$ for each polarization state. 


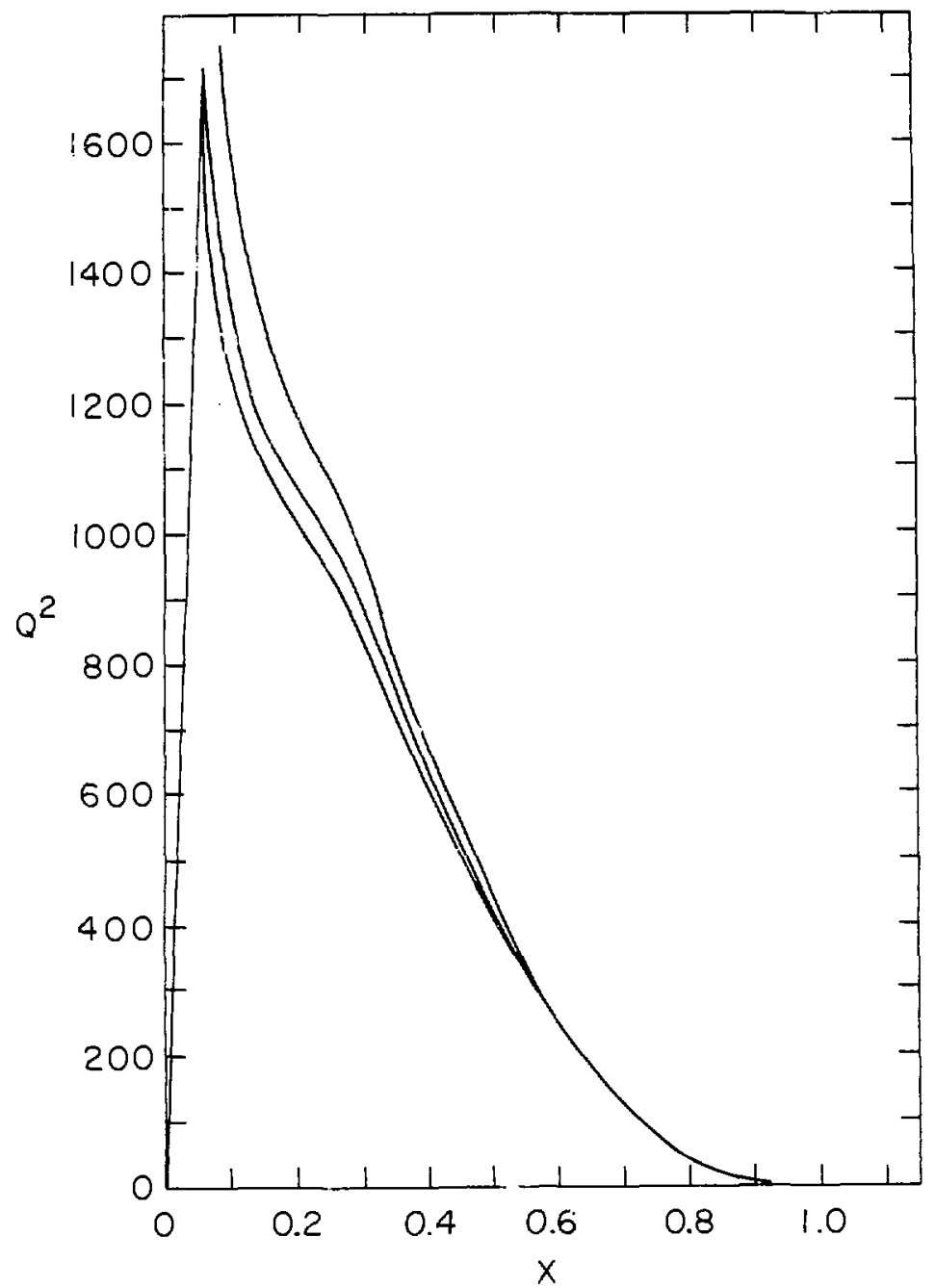

Fig. 2. The luminosity boundary for the reaction $e+p+e^{\prime}+x$ for $s=$ $32,000 \mathrm{GeV}^{2}$, $\sin ^{2} \theta_{\mathrm{w}}=0.22$, and an integrated luminosity of $2 \times 10^{33} \mathrm{~cm}^{-2}$ for each polarization state. 


\section{Structure Functions}

As we have Implied, $F_{2}\left(x, Q^{2}\right)$ is a function that describes the density of partons in $x$, which was considered to be independent of $Q^{2}$ in earlier days. Now we are aware that scale breaking occurs, and a number of authors ${ }^{6}$ have made parametrizations of the quark and gluon densities as a function of $x$. As $Q^{2}$ increases, these densities change, mostly in that the effect of QCD is to take into account the emission of gluons which in turn become quark-antiquark pairs, forming an enhancement of the number of "wee" partons' and a corresponding diminution of the quarks at high $x$. Buras and Gaeners offered an early parametrization followed by some nominally more accurate verstons by Owens and Reya and Baier et al. ${ }^{6}$ In this note, we will follow Buras and Gaeners partly because of the ease of comparison with other authors concerned with e-p. Buras and Gaemers parametrize the distributions as valence quarks, SU(3) symetric sea quarks, charm quark distribution, and a gluon distribution. We will not go into detail here except to say that the valence quark distributions go like

$$
x V \sim x^{n} 1(1-x)^{n} s
$$

and the others like

$$
x S \sim(1-x)^{n_{3}}
$$

The coefficients and the $n$ depend on $Q^{2}$ through a scale breaking paraneter

$$
\bar{s}=\ln \left(\frac{\ln Q^{2} / \Lambda^{2}}{\ln Q_{0}^{2} / \Lambda^{2}}\right)
$$

$\Lambda$ is typically $0.3 \mathrm{GeV}$ and $Q_{0}^{2}=1.3 \mathrm{GeV}^{2}$. The value of $Q_{0}^{2}$ is unimportant but the value of $\Lambda$ is crucial in QCD evaluations.

The $n$ are linear in $\mathbf{s}$. Fits are done to experimental data on leptoproduction to get all the necessary constants. The other authors use a similar technique, but with variations in the parametrization. The figure beiow shows the Buras-Gaemers distributions for two values of $\mathrm{Q}^{2}$ showing the scale breaking effect. 


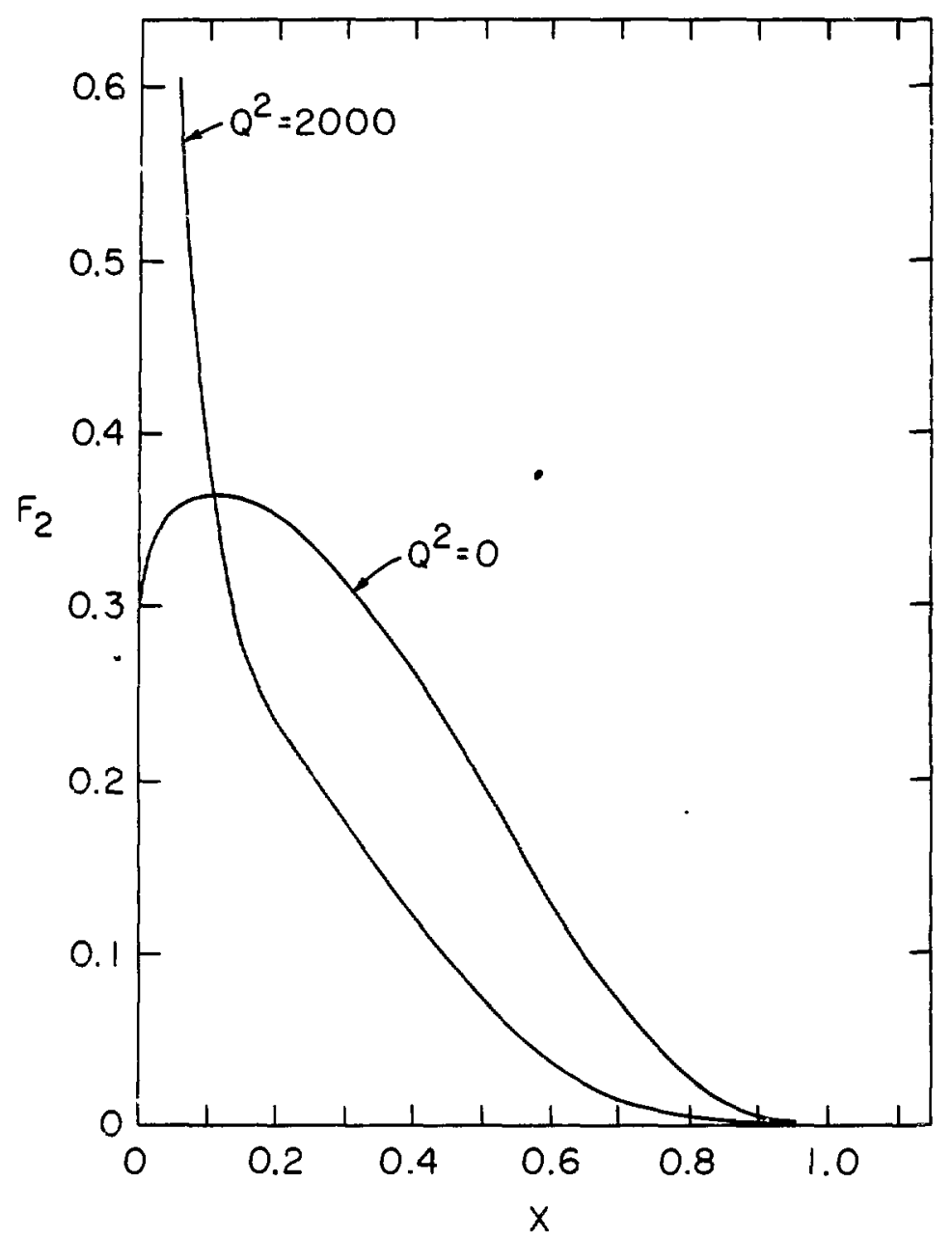




\section{The Charged Current Process}

The charged current cross section is easy to calculate but probably hard to measure. In tems of the usual structure functions, the cross section for the process

$$
e+p+v+x
$$

is written

$$
\frac{d^{2} \sigma}{d x d y}=\frac{G_{F}^{2} s}{\pi}\left[(1-y) F_{2}+y^{2} x_{1}-y(1-y / 2) x F_{3}\right]
$$

We use Callan-Gross

$$
=\frac{G_{F}^{2} s}{2 \pi}\left(\left[1+(1-y)^{2}\right] F_{2}+\left[1-(1-y)^{2}\right] x_{3}\right.
$$

$F_{2}$ and $x F_{3}$ are not the same as in the photon exchange case. For example, an incident $e^{-}$produces a $\mathrm{W}^{-}$only, which is capable of the transitions $u+d$ or $\bar{d} \rightarrow \bar{u}$. If we restrict ourselves to the first two flavors, then in terms of quark densities

$$
F_{2}=(u+c+\bar{d}+\bar{s})
$$

Also

$$
x F_{3}=-(u+c)+(\bar{d}+\bar{s})
$$

We rewrite the cross section in terms of these quark structure functions

$$
\frac{d^{2} \sigma}{d x d y}=\frac{G_{F}^{2}}{2 \pi} 2 s\left((u+c)+(\bar{d}+\bar{s})(1-y)^{2}\right)
$$

then because of $(\mathrm{V}-\mathrm{A})$, only quarks of a particular helicity interact. When this helicity is such as to allow all $\ell_{z}$ of the incident $W$ to 
conserve angular momentum, then there is no $(1-y)^{2}$ term. Then the other helicity occurs, then the electron may not scatter at $\theta=\pi, y=1$ from angular momentum considerations. Then in our preferred variables

$$
\frac{d^{2} \sigma}{d x d Q^{2}}=-\frac{G_{F}^{2}}{\pi} \frac{1}{x}\left((u+c)+(1-y)^{2}(d+\bar{s})\right)
$$

and in our usual bin structure

$$
\operatorname{RATE}=\frac{G_{\mathrm{F}}^{2}}{\pi} Q^{2}\left((u+c)+(1-y)^{2}(\bar{d}+\bar{s})\right) \frac{d Q^{2}}{Q^{2}} \frac{d x}{x},
$$

and numerically the yield is shown in Figure 3 for our usual luminosity and s. Notice that $s$ does not come into this expression (against intuition), but if we assure that the structure functions do not vary with $Q^{2}$, and we Integrate over all $Q^{2}$, then

$$
\text { RATE } \sim \int \mathrm{dQ}^{2} \frac{\mathrm{dx}}{\mathrm{x}}=\mathrm{sd} \mathrm{x}
$$

For a given $x$ bin, we have a rate that is proportional to s. Again if we are interested in distributions, we do not improve the situation in any single bin by improving $s$, but, of course, we do get an increased range and the bin content is higher at higher $Q^{2}$. The expression above ignores the effect of the $W$ mass, meaning that we should insert a $W$ propagator, so

$$
\frac{d^{2} \sigma}{d x d Q^{2}}=\frac{G_{F}^{2}}{\pi} \frac{1}{x} \frac{Q^{2}}{\left(1+Q^{2} / M_{v}^{2}\right)^{2}}\left((u+c)+(1-y)^{2}(d+\vec{s})\right)
$$

and the effect of the propagator can be seen in the non-linear variation of rate with $Q^{2}$ in Figure 3 . 


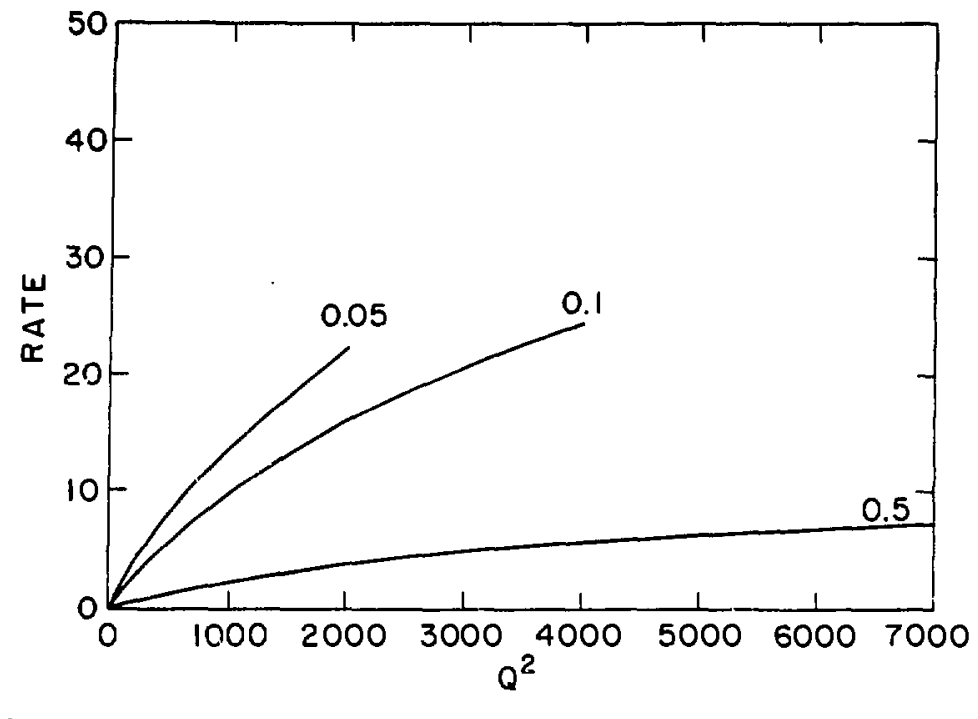

Figure 3. The rate for the charged current reaction $e+p+v+x$ for $s$ $=40,000$ and $\sin ^{2} \theta_{\omega}=0.22$.

The handy rule of thumb for rate in a bin is

$$
R A T E=R_{0} \frac{Q^{2}}{\left(1+Q^{2} / M_{w}^{2}\right)^{2}} \frac{d^{2}}{Q^{2}} \frac{d x}{x} .
$$


Then, for our usual bin widths, $R_{0}$ for various values of $x$ are

$\begin{array}{ll}x & R_{0} \\ 0.05 & 2 \\ 0.1 & 1.5 \\ 0.25 & 1 \\ 0.5 & 0.3\end{array}$

For an overall rate, we can integrate the expression over $Q^{2}$ and approximate the $x$ distribution by assuming that 1 is flat out to $x_{\max }$ $(0.3)$, say, and zero thereafter:

$$
\operatorname{RATE}=\left\langle R_{0}\right\rangle M_{w} \ln \left(1+\frac{s x_{\text {max }}}{M_{w}^{2}}\right) .
$$

This is within $20 \%$ of the exact calçulation with $\left\langle R^{\circ}\right\rangle=1.5$ and $x_{\max }$ $=0.25$. Notice that when $s x_{\max }>\mathrm{M}^{2}$, the effect of increasing $s$ is much reduced. When $3 x_{\max }=\mathrm{M}_{\mathrm{w}}^{2}$, and for $10^{38}$ total luminosity, we have $10^{4}$ events, the error in $M_{w}$ is twice that of the rate itself, so we might (very naively) say that this length of run gives $\Delta M_{y j}$ of $2 \%$. Real calculations with resolution etc. belleve that the error would be twice this.

Implicit in the formulas of this section is that the electrons are left-handed, the simple nodel assumes that in the right-handed positron case, int are exchanged and similar arguments give the cross section

$$
\frac{d^{2} \sigma}{d x d Q^{2}}=\frac{G_{F}^{2}}{\pi} \frac{Q^{2}}{\left(1+Q^{2} / M_{W}^{2}\right)^{2}}\left((\bar{u}+\bar{c})+(1-y)^{2}(d+s)\right) \frac{d Q^{2}}{Q^{2}} \frac{d x}{x} .
$$

The weak neutral current is sensitive to the coupling of the $z^{\circ}$ to the different quarks. We can in principle explore the possibilities of more than one $Z^{\circ}$ and couplings other than those of the standard model. However, in the spirit of providing a base for comparison with exotic models, we will outline the rate calculations from the standard model in some detail. Moreover, it is convenient to calculate a factor describing the effect on the one-photon exchange cross section and to use the results of the previous sections to make the neutral current effect clearer. 
The cross section can be written as the sum of three terms; the single photon part, the weak part, and the interference part. In practice, the weak part is generally negligible, and it is only the interference part that is important in the e-p colliders that have been considered. We write the cross section as follows:

where

$$
\frac{d \sigma}{d x d Q^{2}}=\left.\frac{d \sigma}{d x d Q^{2}}\right|_{Y} F /(f+F \underset{2 e m}{)}
$$

$$
F=\sum_{i j} G_{\eta}^{j} p^{i} p^{j}\left(f_{+}+F_{2}^{i j}+\xi_{\eta} f_{-} \times F_{3}^{i j}\right)
$$

for standard model $1, j=1,2$, and the subscript $n$ refers to the incoming lepton state. When $1=j=1$, we have the photon part and $F=1$. When 1 and $j$ are both 2 , we have the weak part, and when the 1,2 combinations occur, we have the interference term.

The $G_{n}$ refers to the couplings of the electron to the photon or $z^{0}$, and $n$ runs 1 through 4 for $e_{L}^{-}, e_{\bar{R}}^{-}, e_{L}^{+}, e_{R^{*}}^{+}$Then

$$
\begin{gathered}
G_{n}^{1}=-1,-1,-1,-1, \\
G_{n}^{2}=\left(-1+2 \sin ^{2} \theta_{w}\right), 2 \sin ^{2} \theta_{w}, 2 \sin ^{2} \theta_{w},\left(-1+2 \sin ^{2} \theta_{w}\right), \\
\xi=+1,-1,+1,-1 .
\end{gathered}
$$

The $\mathrm{p}^{1}$ are propagator terms

$$
\begin{aligned}
& p^{1}=1 \\
& p^{2}=\sqrt{ } 2 \frac{G_{F} Q^{2} M_{Z}^{2}}{e^{2}\left(M_{Z}^{2}+Q^{2}\right)},
\end{aligned}
$$

where $\mathrm{M}_{Z}=37.4 / \sin \theta_{\mathrm{W}} / \cos \theta_{\mathrm{W}}$ in the standard model. The kinematic terms are given as 


$$
\begin{aligned}
& f_{+}=\frac{1}{2}\left[1+(1-y)^{2}\right] \\
& f_{-}=\frac{1}{2}\left[1-(1-y)^{2}\right] .
\end{aligned}
$$

The structure functions $F_{2}^{11}=F_{2}$ and $x F_{3}^{11}=0$.

$$
\begin{aligned}
F_{2}^{12} & =\sum_{q}\left(G_{q L}^{1} G_{q L}^{2}+G_{q R}^{1} G_{q R}^{2}\right)(q+\bar{q}) \\
x F_{3}^{12} & =\sum_{q}\left(G_{q L}^{1} G_{q L}^{2}-G_{q R}^{2} G_{q R}^{2}\right)(q-\bar{q}) .
\end{aligned}
$$

The coupling of the $\gamma$ to the quarks is proportional to the charge $Q_{q}$. The coupling of the $Z$ to the quarks is given by the standard model.

$$
\begin{aligned}
& \mathrm{G}_{\mathrm{q}}^{1} \quad-1 / 3 \\
& -1 / 3 \\
& \begin{array}{ll}
u & c \\
d & s
\end{array} \\
& G_{G_{L}}^{2} \quad 1-4+2 / 3 \sin ^{2} \theta w \\
& 1-4 / 3 \sin ^{2} \theta_{w} \\
& \text { u c } \\
& -1+2 / 3 \sin ^{2} \theta_{\mathrm{w}} \\
& \text { d } s \\
& G^{2} \quad-4 / 3 \sin ^{2} \theta_{w} \\
& q_{R} \quad 2 / 3 \sin ^{2} \theta_{w} \\
& \begin{array}{r}
-4 / 3 \sin ^{2} \theta \\
2 / 3 \sin ^{2} \theta
\end{array} \\
& \text { u c } \\
& \text { d } s
\end{aligned}
$$

These couplings allows us to evaluate $F$ for any incoming lepton state and any quark $q$ or $\bar{q}$. We use the structure functions of the previous section to evaluate $q\left(x, Q^{2}\right)$.

Nunerically, the coefficient of $\mathrm{p}^{1} \mathrm{p}^{2}$ is $\mathrm{\prime}^{\prime} 2 \mathrm{G}_{\mathrm{F}} / \mathrm{e}^{2}=1.75 \times 10^{-4} \mathrm{GeV}^{-2}$ and for $Q^{2} \sim 1000$ and below, this term is snall compared to 1 , and so the weak neutral current is proportional to $G_{F}^{2}$ squared and is negligible. 
For $Q^{2}$ of this order, $Q^{2} \ll m_{2}^{2}$, and the propagator effect is small. Also, $f_{\text {- }}$ is small. It is also true that in crude terms, the u valence quark dominates $q$ and $\bar{q}$ is negliglble.

The factor for $e_{\bar{L}}^{-}$is then approximately $\sim 1+2\left(1-2 \sin ^{2} \theta_{w}\right) \times 1.8$ $\times 10^{-4} \times Q^{2} \times 2 / 3\left(1-4 / 3 \sin ^{2} \theta_{w}\right)=1.10 Q^{2}$. This compares with 1.08 when it is done properly. of course, the effect is maximally destructive for $e_{L}^{+}$when $G_{\eta}^{2}$ effectively changes sign. The important facts to remember are that the interference part of WNC for $e_{\mathrm{L}}^{-}$is roughly proportional to $Q^{2}$ and is about $10 \%$ at $Q^{2}$ of 1000 . We show a spectfic calculation in Figure 4. The reason that the curves show a slight tendency to tncrease in slope is the scale breaking effect that more parton density occurs at low $x$ as $Q^{2}$ Increases with a consequent increase in the WNC offsetting the propagator.

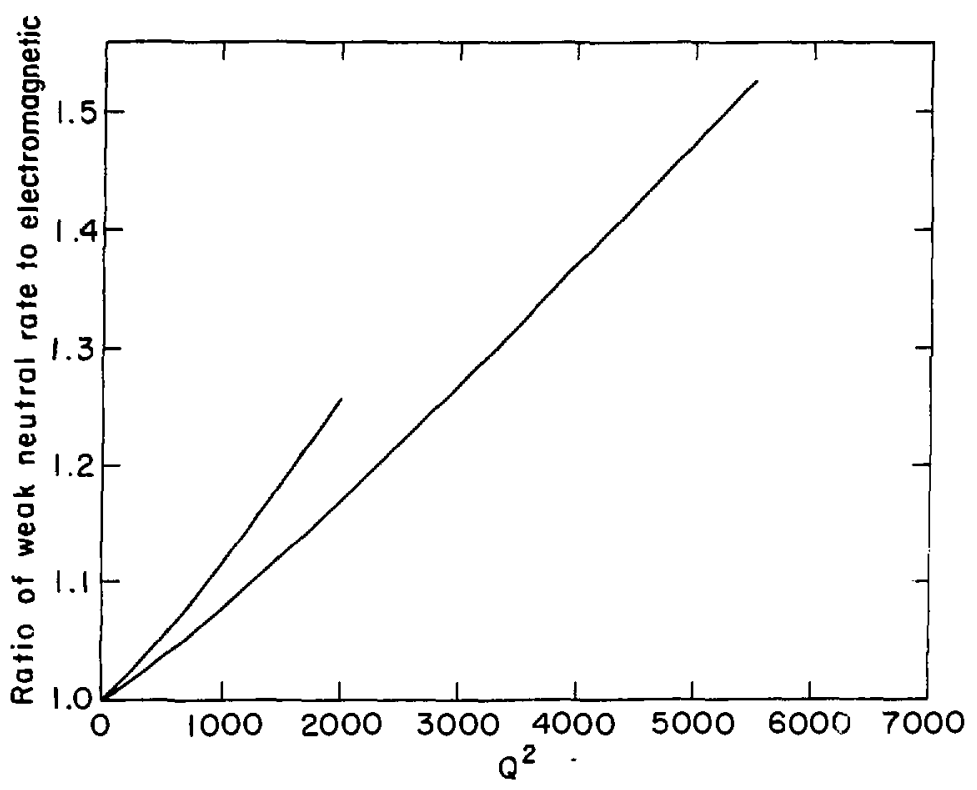

Figure 4. Ratio of the weak neutral current cross section to the electrmagnetic cross section as a function of $Q^{2}$ for $x=0.05$ and $x=0.5$. 


\section{Photoproduction}

Vector meson production has been studied in detail using photon beams and at low $Q^{2}$ where clean signals are available in diffractive production of vector mesons. In an e-p collider, quasi-photoproduction can be studied at very high equivalent photon eriergies utilizing the low $\mathrm{Q}^{2}\left(<1 \mathrm{GeV}^{2}\right)$ portion of the cross section. The photon energy (E- $\left.\mathrm{E}^{\prime}\right)$ should be multipiled by the $\gamma$ of the proton $\left(\mathrm{E}_{\mathrm{p} / \mathrm{m}_{\mathrm{p}}}\right)$ to get the equivalent photon energy for the proton rest syster. In the case of the $20 \times 400$ collider $4 \mathrm{TeV}$ equivalent photon energy is eastly avallable. The $20 \mathrm{x}$ 400 effective luminosity is given by the Welzacker-Willians expression

$$
\mathrm{d}^{2} \mathrm{~L}_{\gamma^{*}}=\frac{\alpha}{2 \pi} \mathrm{L}_{\text {ep }} \frac{\mathrm{dQ}^{2}}{\mathrm{Q}^{2}} \cdot \frac{\mathrm{dy}}{\mathrm{y} \cdot}\left[1+(1-\mathrm{y})^{2}\right]
$$

This luminosity is dominated by the low $\mathrm{Q}^{2}$ region, and providing $\mathrm{y}$ is large enough that we are above threshold, by the low $y$ region also. We can divide the rate calculations into two parts, the virtual photon flux described above and the production cross section for heavy quark states.

$$
\sigma^{2} L_{\gamma^{*}}=\sigma_{0} \cdot\left(\frac{\mathrm{m}_{\mathrm{v}}^{2}}{\mathrm{~m}_{\mathrm{v}}^{2}+\mathrm{Q}^{2}}\right)^{2} \cdot\left(1-\mathrm{y}_{\mathrm{th}} / \mathrm{y}\right) \text {. }
$$

The first term is a consequence of vector dominance and has been measured for $\rho, \phi$ and $J / x$. The second term appears to $f$ it the observed $Q^{2}$ dependence for all vector meson production from $\rho$ to $J / x$. The third term is a threshold factor, proportional to the square of the vector meson morentum in $c . m$. of the vector meson-proton system.

$\sigma_{o}$ is proportional to $\left(Q_{q} / m_{q}\right)^{2}$ and is approximately $0.5 \mu \mathrm{b}$ for charm production, and $10 \mu \mathrm{b}$ for the 1 ight quarks $\left(\rho^{\circ}\right)$.

At. low $Q^{2}$ we can write

$$
\mathrm{y} \sim\left(1-\frac{E^{\prime}}{\mathrm{E}}\right)
$$

and since the energy squared in the c.m. of the virtual photon proton system is

$$
w^{2}=-Q^{2}+s y+m_{p}^{2},
$$


at low $Q^{2}$ again

$$
y_{t h}=\frac{m_{v}}{s}\left(m_{v}+2 m_{p}\right)
$$

For any reasonable rate this is a small number, for example, at $40 \mathrm{GeV}$ $y_{\text {th }} \sim 0.05$ on a $20 \times 400$ collider.

The minimum $Q^{2}$ is usually given by the minimum tagging angle for the outgoing electron $\theta$, then for small

$$
Q_{m 1 n}^{2}=E^{2}{ }^{2}(1-y)
$$

when $\theta=0$, the minimum value for no $\operatorname{tag}$ is $\mathrm{m}^{2} \mathrm{y}^{2} /(1-y)$.

The yield can now be calculated

$$
\begin{aligned}
& \text { Yield }=\frac{\alpha}{2 \pi} \quad L_{e p} \cdot \gamma_{0} \int_{y_{t h}}^{1} \frac{d y}{y}\left(1-(1-y)^{2}\right)\left(1-\frac{m_{v}^{2}}{1-y}\right) \\
& \int_{Q_{m i n}^{2}}^{Q_{\max }^{2}} \frac{d Q}{Q^{2}}\left(\frac{m_{v}^{2}}{\left(m_{v}^{2}+Q^{2}\right)}\right)^{2} .
\end{aligned}
$$

The yield is doninated by low $Q^{2}$ as we have sald before, and then the second part of the integral is approximate $1 \mathrm{y} \ln \left[\mathrm{m}_{\mathrm{e}}^{2} / \mathrm{E}^{2} \theta^{2}(1-\mathrm{y})-1\right]$ and the first part is $\ln \left(\sin _{v}^{2}-1\right)$.

It is important then to keep the tagging angle small, and $s \gg m_{v}^{2}$, althoigh the improvement in the photon flux is only logarithmic with $s / m_{v}^{2}$.

For an example, take $40 \mathrm{GeV}$ top in a $20 \times 400$ collider. The value of $\sigma_{0}$ is $3 \mathrm{nb}$, and with a tagging angle of $3^{\circ}$ the flux integral is 5.2 . The yield for an e-p luninosity of $10^{38}$ is $1.8 \mathrm{~K}$ events. Without tag the yield increases by a factor of about 3 . 


\section{References}

1. Cheep; an e-p Facility in the SPS. CERN 78-02.

2. Proceedings of the Study of an ep Facility for Europe. U. Amaldi, Editor. DESY 79/48, August 1979.

3. CHEER; Canadian High Energy Electron Ring. A Feasibility Study for an Electron Ring at a High Energy Proton Accelerator, Richard J. Hemingway, September 1980.

4. Electron-Proton Interaction Experiment. Fermilab Proposal 659, June 1981. Spokesmen: W. Lee, R.R. Wilson.

5. Feasibility of an e-p Facility as Phase 1 for a BNL Colliding Bear Accelerator, Working Group Report, K. Johnsen, Chairman, June 1982.

5. A.J. Buras and K.J.F. Gaemers. Nuclear Physics B132, 249 (1978). J.F. Owens and E. Reya, Phys. Rev. D17, 3003. R. Baier et al, Z Physik C2, 265 (1979).

7. D.G. Cassel et a1, Phys, Rev. D., 24, No. 1, 2787 (1981). 


\section{Acknowledgements}

It is a pleasure to acknowledge the leadership of Kjell Johnsen in the e-p study group at Brookhaven from which this note stems. It is also a pleasure to record the patience with which Larry Trueman helped me with my misconceptions, and many helpful comments from Frank Taylor. Jim Wiss furnished the outline of the photoproduction section. 